\title{
Cholinesterase inhibitors for gait, balance, and fall in Parkinson disease: a meta-analysis
}

\author{
Jia-Hung Chen ${ }^{1}$, Tsai-Wei Huang $\mathbb{1}^{2,3,4}$ and Chien-Tai Hong ${ }^{1,5 凶}$
}

Gait disturbance and imbalance are the major symptoms of Parkinson disease (PD), with fall being the most undesirable consequence. However, few effective evidence-based treatments are available for alleviating these symptoms and preventing falls. Cholinesterase inhibitors (ChEls) are a well-established treatment for PD dementia with possible impacts on gait, balance, and fall reduction. The present study involved a meta-analysis of randomized controlled trials (RCTs) to investigate the effects of ChEls on gait, balance, and fall in patients with PD. We searched for studies using the PubMed, Embase, and Web of Science databases. The major outcomes were effects on gait parameters, balance, and fall. This study was registered with PROSPERO (CRD42021254733). Five RCTs were included in the present meta-analysis. ChEls did not significantly increase gait speed in PD patients (mean difference [MD]: $0.03 \mathrm{~m} / \mathrm{s}, 95 \%$ confidence interval [Cl]: -0.02 to $0.07, p=0.29$ ). However, ChEl treatment significantly decreased step or stride variability during the single task (standard $\mathrm{MD}$ : $-0.43,95 \% \mathrm{Cl}=-0.79$ to $-0.06, p=0.02$ ). Regarding fall and balance, trending but nonsignificant beneficial effects were observed with ChEl treatment. In conclusion, although ChEl treatment did not significantly improve gait speed and reduce fall, it can significantly reduce step or stride variability. Considering that gait disorder is a challenging issue in patients with PD and that ChEls are generally tolerable, the present meta-analysis may provide more evidence for the benefit of ChEls on PD gait disturbance as an alternative treatment consideration.

npj Parkinson's Disease (2021)7:103; https://doi.org/10.1038/s41531-021-00251-1

\section{INTRODUCTION}

Parkinson disease (PD) is the second most common neurodegenerative disease ${ }^{1}$. The cardinal motor symptoms of PD include rigidity, tremor, bradykinesia, and gait disturbance. Unlike the first three symptoms, gait disturbance does not respond well to dopaminergic replacement therapy ${ }^{2}$. The postural instability and gait disturbance subtype of PD deteriorated rapidly compared with the other subtypes ${ }^{3}$. Traditional subthalamic deep brain stimulation usually does not alleviate gait disturbance and sometimes may even worsen it ${ }^{4}$. The most devastating consequence of gait disturbance and imbalance is fall, which results in fracture, traumatic brain injury, and fear of walking. Gait disturbance and fall are substantial threats to the quality of life and mortality of individuals with $\mathrm{PD}^{5}$.

The risk factors for gait disturbance and falls in PD are variable. It could be age-related, such as anxiety, depression, osteoporosis, polypharmacy, weakness, or due to PD-related symptoms such as rigidity, cognitive impairment, dyskinesia, postural instability, and slow mobility ${ }^{6}$. In people with PD, cognitive dysfunction is a major contributor to gait disturbance and fall, especially the most detrimental form of gait disturbance, that is, freezing of gait $(\mathrm{FOG})^{7,8}$. The signature manifestation of cognitive dysfunctionrelated FOG is a remarkable worsening of gait during dual-task challenges, which are usually a gait test accompanied by a calculation ${ }^{9}$.

Evidence of cognitive contributions to gait and fall are wellacknowledged, but the precise mechanisms are not completely understood. In those people with dementia, gait impairments are more prevalent than in normal aging and are related to the severity of cognitive impairment ${ }^{10}$. Similar results are also demonstrated in risk of falls that older adults with moderate to the severe cognitive impairment have a higher risk ${ }^{11}$. Attention and executive function are thought to be responsible for postural and gait stability ${ }^{12,13}$. According to the pre-clinical studies, cortical cholinergic neurons-lesioned parkinsonism mice demonstrated great gait disturbance and fall propensity ${ }^{14}$, which is suspected to result from the disruption of cortical processing of task and movement cues for the striatum ${ }^{15}$. In addition, augmentation of cholinergic effect by either rivastigmine or donepezil partially attenuated the symptoms in this dual-lesioned model ${ }^{16,17}$. More than cognitive dysfunction, degeneration of the brainstem cholinergic neurons, pedunculopontine nucleus (PPN), and laterodorsal tegmental nucleus (LDT) are also proposed as one of causative $t$ for gait disturbance in individuals with $\mathrm{PD}^{18}$.

Cholinesterase inhibitors (ChEls) are widely used for the management of dementia, including Alzheimer disease and vascular dementia. Regarding PD dementia, at present, only rivastigmine provides a significant evidence-based therapeutic effect $^{15}$. Numerous studies have examined the effects of ChEls on patients with PD, but most have focused on cognition and dementia ${ }^{19-22}$. Some pre-clinical studies investigated the impact of gait, balance, and fall and demonstrated possible benefits ${ }^{16,17}$. However, most were small-scale clinical studies, providing low evidence for benefits. The present study aimed to summarize the effect of ChEls on gait, balance and falls in patients with PD.

\section{RESULTS}

Summary of the included studies

Figure 1 displays the study selection flowchart. Our initial search yielded 336 studies, 204 of which were eliminated due to duplication. The remaining 132 studies were subjected to title

\footnotetext{
${ }^{1}$ Department of Neurology, Shuang-Ho Hospital, Taipei Medical University, New Taipei, Taiwan. ${ }^{2}$ Cochrane Taiwan, Taipei Medical University, Taipei, Taiwan. ${ }^{3}$ School of Nursing College of Nursing, Taipei Medical University, Taipei, Taiwan. ${ }^{4}$ Center for Nursing and Healthcare Research in Clinical Practice Application, Wan Fang Hospital, Taipei Medical University, Taipei, Taiwan. ${ }^{5}$ Department of Neurology, School of Medicine, College of Medicine, Taipei Medical University, Taipei, Taiwan. ${ }^{凶}$ email: ct.hong@tmu.edu.tw
} 


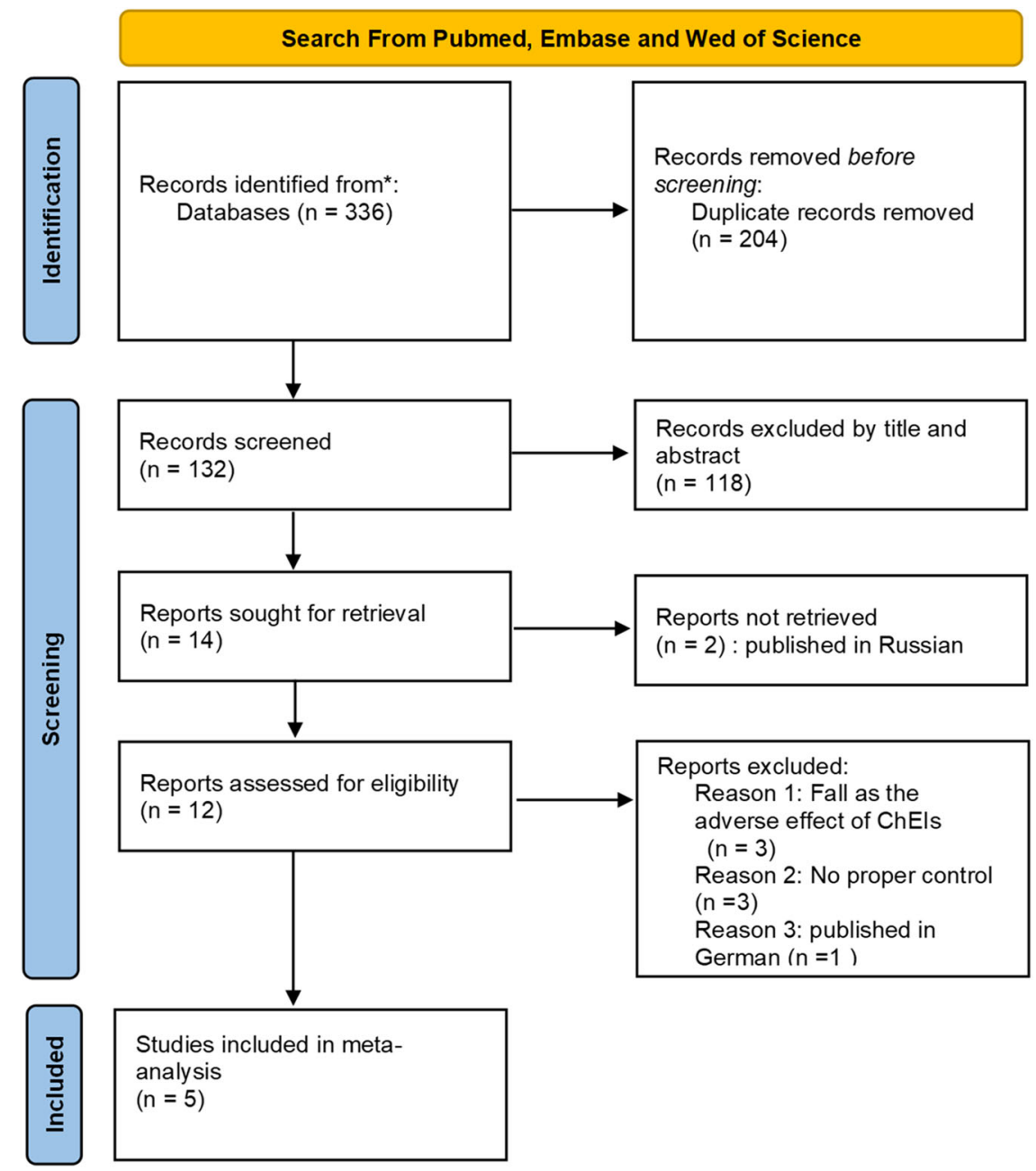

Fig. 1 The study selection flowchart. Randomized controlled trials published before May 2021 in the PubMed, Embase, and Web of Science databases were searched following the Preferred Reporting Items for Systematic Review and Meta-Analysis (PRISMA) guidelines.

and abstract screening, and 118 were excluded. The final 12 studies, except two that were published in Russian, were entered in the full text review. Three studies were excluded because fall was the adverse effect of ChEl treatment and not the major outcome, and another three studies were excluded due to the lack of a placebo control. One study was excluded as it was published in German. The remaining five eligible RCTs ${ }^{23-27}$ were included in our analysis. These RCTs were published between 2010 and 2020 and had sample sizes ranging from 20 to 130 . The mean age of patients was approximately 70 years, and all study cohorts had male preponderance. All five studies recruited patients diagnosed as having idiopathic PD. Two studies specifically enrolled patients with a history of fall. Rivastigmine was prescribed in two studies, and in the remaining three, donepezil was prescribed (Table 1). All of them were categorized into low risk or some concerns regarding the risk of bias (Table 2).

\section{Effects on gait}

Our meta-analysis of gait parameters revealed that ChEls did not significantly increase gait speed in patients with PD (MD: $0.03 \mathrm{~m} / \mathrm{s}$, $95 \% \mathrm{Cl}:-0.02$ to $0.07, p=0.29$ ). The heterogeneity of this analysis was nonsignificant $\left(I^{2}=15 \%\right.$; Fig. $\left.2 a\right)$. By contrast, a significant beneficial effect on step or stride variability during the single task (SMD: $-0.43,95 \% \mathrm{Cl}:-0.79$ to $-0.06, p=0.02$ ) was demonstrated. The heterogeneity of this analysis was nonsignificant, with $l^{2}$ being $48 \%$ (Fig. 2b).

\section{Effects on balance and fall}

Regarding balance and fall, trending but nonsignificant beneficial effects were conferred. Two studies were added into the quantitative analysis for balance improvement, which revealed highly heterogenous $\left(I^{2}=68 \%\right)$ and nonsignificant results of balance improvement (SMD: 1.96, 95\% Cl: -2.22 to $6.14, p=$ 0.36; Fig. 3a). Regarding fall reduction, two studies demonstrated highly variable numbers of falls per year, which leads to the high heterogenicity of the effect size $\left(I^{2}=92 \%\right)$. The effect of ChEls on fall reduction was not significant (SMD: $-0.82,95 \% \mathrm{Cl}:-1.85$ to $0.21, p=0.12$ ).

\section{DISCUSSION}

We summarized the results of five RCTs published between 2010 and 2020. All studies recruited patients diagnosed as having idiopathic PD by neurologists. The present study demonstrated that although ChEls did not significantly increase gait speed in individuals with PD, step or stride variability significantly improved. Treatment with ChEls provided trending improvements in fall prevention and balance, but the results were highly variable. Considering that patients with PD generally tolerated ChEls, they were a possible treatment alternative for gait disturbance for these patients.

Gait is a complex task involving the cognitive, sensorimotor, and cerebellar systems ${ }^{28}$. The cholinergic transmission is vital in 


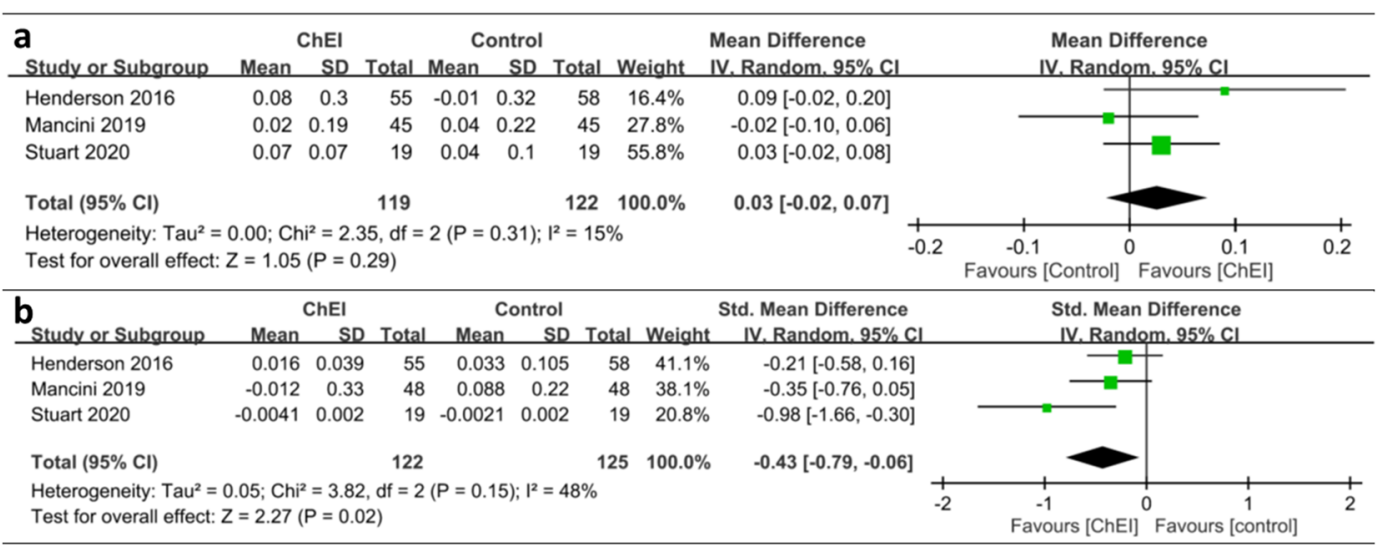

Fig. 2 Effect of cholinesterase inhibitors (ChEls) on the gait speed and step/stride variability of patients with Parkinson disease (PD). a ChEls did not significantly increase gait speed in patients with PD (Mean Difference [MD]: $0.03 \mathrm{~m} / \mathrm{s}, 95 \%$ confidence interval [Cl]: -0.02 to $0.07, p=0.29$ ). b A significant beneficial effect on step or stride variability during the single task (Standardized MD [SMD]: $-0.43,95 \% \mathrm{Cl}$ : -0.79 to $-0.06, p=0.02$ ) was demonstrated.

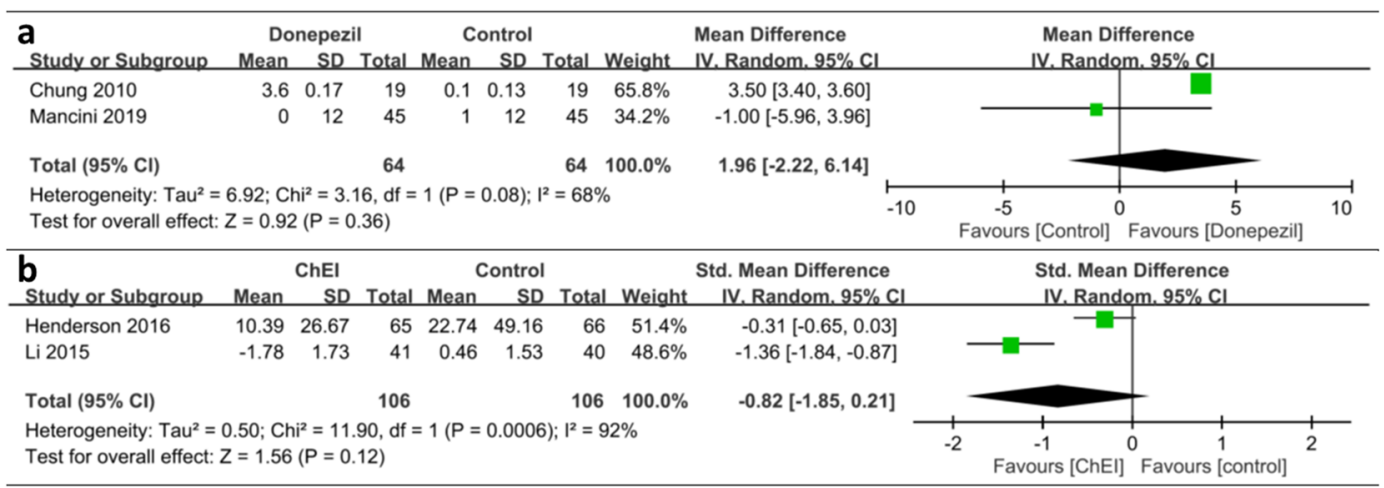

Fig. 3 Effect of cholinesterase inhibitors (ChEls) on the balance and fall frequency of patients with Parkinson disease (PD). a Trending but nonsignificant beneficial effects were conferred for balance improvement (Standardized Mean Difference [SMD]: 1.96, 95\% Confidence Interval $[\mathrm{Cl}]$ : -2.22 to $6.14, p=0.36$ ). $\mathbf{b}$ The effect of ChEls on fall reduction was not significant (SMD: $-0.82,95 \% \mathrm{Cl}:-1.85$ to $0.21, p=0.12$ ).

performing this task modulation of cognition and motor systems ${ }^{18}$, since the basal forebrain cholinergic nuclei, the nucleus basalis of Meynert (NBM), and the substantia innominata project to the cerebral cortex, thalamus, and striatum. PD dementia is associated with NBM degeneration ${ }^{29}$, and patients with PD dementia converted from PD-related mild cognitive impairment exhibited more significant loss of the $\mathrm{NBM}^{30}$, which may be responsible for worse gait performance. In people with PD, cognitive impairment in PD also limits the execution of complex tasks, such as gait. The severity of PD-FOG, the most devastating gait disturbance in PD, is inversely associated with the set-shifting performance. For those with PD, their dual-task (simultaneous cognitive load) walk test demonstrated low gait speed ${ }^{31}$, increased gait variability ${ }^{32}$, and poor coordination ${ }^{33}$. ChEls improve cognition in patients with PD dementia through the cholinergic augmentation effect ${ }^{19}$, which may further improve gait disturbance. The cholinergic system is also present in the brainstem PPN and LDT that control the gait performance. A lesion in the PPN impaired motor coordination in experimental animals ${ }^{34}$, and the loss of cholinergic neurons in the PPN was correlated with falls in people with $\mathrm{PD}^{35}$. In addition, the thalamus is a projection of the PPN, and the reduction of thalamic cholinergic projection was greater in those with PD-related falls ${ }^{36}$. Central-acting ChEls enhance brain cholinergic activity, modulating gait through cognitive and motor coordination aspects.

Step or stride variability is a cardinal feature of gait disorders in PD and is distinctly affected by stride length and frequency ${ }^{37}$. People with PD usually lost the ability to regulate the stride-to- stride fluctuations while walking, especially in those with $\mathrm{FOG}^{38}$. Disability in stride variability is also considered a risk factor of cognitive decline ${ }^{39}$, and the relationship between step time variability and falls has been identified ${ }^{40}$. The present study demonstrated that ChEls improved step or stride variability in patients with PD, both of which are highly associated with cognition ${ }^{41}$. This effect was observed in all included studies, especially that conducted by Stuart et al. ${ }^{27}$. On the other hand, only an increasing trending in gait speed was observed upon ChEl treatment. The slowness of gait in PD may result from bradykinesia, FOG, and hesitation. The latter two conditions may be triggered by anxiety or fear of fall, and they were more responsive to anxiolytic agents than $\mathrm{ChEls}^{42}$.

Balance control on movement (dynamic movement) and stillness (static balance) requires integrating all levels of the nervous system. The basal ganglia are involved in controlling balance via the thalamic-cortical-spinal loops and via the brainstem PPN, cholinergic neurons, and the reticulospinal system ${ }^{43}$. For patients with PD, rigidity, bradykinesia, impaired sensory integration, and cognitive impairment contribute to imbalance, and the latter two factors are dopamine insensitive ${ }^{44}$. Poorer balance was associated with the PPN-thalamic cholinergic system $^{35}$, and the PPN deep brain stimulation may confer beneficial effects on balance in patients with $\mathrm{PD}^{45}$. The present meta-analysis was on balance from two RCTs ${ }^{23,24}$, and the improvement was only marginal. Another RCT conducted by Handerson et al. ${ }^{26}$ reported a significant benefit of rivastigmine on balance, assessed by controlled leaning balance. However, the 
authors presented only the categorical outcome of the tests as low, medium, high, and very high, which made a quantitative meta-analysis impossible.

Furthermore, Chung et al. $^{23}$ revealed inconsistent results. However, it demonstrated the relative benefit of donepezil on balance assessed by the Activities of Balance Confidence, but no difference was noted when the Berg Balance Scale was used. Throughout the present meta-analysis, a significant benefit of ChEls on balance in patients with PD was not demonstrated.

Falls are common among people with PD, and some have recurrent falls ${ }^{46}$. The risk factors of fall in PD can be categorized into generic (i.e., old age, osteoporosis, vertigo, or anxiety) and PDrelated aspects (axial rigidity, cognitive impairment, medications, or $\mathrm{FOG})^{6}$. ChEl treatment was speculated to alleviate cognitive impairment and FOG; however, it is not effective against other factors. The present analysis included two studies on treatment with rivastigmine, demonstrating a trending but nonsignificant effect on fall prevention. One of the studies conducted by $\mathrm{Li}$ et al. $^{25}$ recruited PD patients with different cognition statuses (normal, $\mathrm{MCl}$, or dementia) without mentioning previous fall history and found a significant reduction in the number of falls compared with the placebo group $(1.82 \pm 1.99$ versus $4.26 \pm 1.63$ per year, $p<0.01)$. The rivastigmine group also had a better cognitive performance at study completion.

On the other hand, Handerson et al. ${ }^{26}$ recruited PD patients with at least one fall, the strongest fall predictor, in the previous year. At baseline, rivastigmine and placebo groups exhibited nonsignificant differences in the number of falls (approximately five falls in the previous year). At study completion, the rivastigmine group had $1.4 \pm 2.47$ falls per month (equal to $16.8 \pm 29.64$ per year) compared with $2.4 \pm 4.40$ falls per month (equal to $28.8 \pm 52.8$ per year) in the placebo group. Although a significant difference was noted in the number of falls between groups, both groups showed a remarkable increase in the number of falls compared with baseline. The number of falls was greater than that reported by $\mathrm{Li}$ et al. ${ }^{25}$. Because of the highly variable number of falls, the present meta-analysis failed to demonstrate significant fall prevention from the two notable results. In addition, one study included in the meta-analysis, conducted by Chung et al. ${ }^{23}$. investigated the number of falls in the placebo and donepezil groups. They found that fall frequency per day in the placebo group was 0.25 (equal to 91.25 per year) compared with 0.13 in the group (equal to 47.45 per year) taking donepezil ( $p<$ 0.05 ). However, that study did not provide information on the baseline number of falls, which limits the utility of its inclusion into a quantitative meta-analysis.

In summary, three studies reported significant fall prevention associated with $\mathrm{ChEl}$ treatment in PD patients. However, a considerable difference in the number of falls per year (1.82-91.25) between the three studies rendered it difficult to confirm a significant and homogenous result from the metaanalysis. We argued that ChEls might prevent falls in PD patients; however, further studies are required to clarify the benefit among a specific group of people with PD.

The strength of the present study was that it analyzed not only fall but also gait parameters and balance. Although not all five studies had complete information, with the help of a metaanalysis, we identified the significant benefit of the ChEls rivastigmine and donepezil on gait variability and the trending benefit on gait speed and fall reduction. The discrepancy of the response upon ChEl treatment among the gait parameters and falls is noted. The possible explanation is that the gait variability is highly cognition-depended ${ }^{47}$, which indicated greater contribution of cholinergic system. On the other hand, gait speed, balance, and fall are affected by the combination of multiple neurotransmitters systems, and the augmentation of single system cannot result in significant impact ${ }^{48}$. Considering that gait disturbance is challenging in the management of PD, a tolerable and possibly effective treatment involving ChEls may be considered. Rivastigmine, which is effective for PD dementia management ${ }^{49}$, is the preferred treatment of choice with dual benefits for people with PD. However, the adverse effect of rivastigmine, such as nausea, vomiting, and tremor aggravation ${ }^{19}$, should be cautiously monitored. Further RCTs are warranted to clearly delineate the cognition-based and non-cognition-based therapeutic benefits of ChEls on PD.

This study had some limitations, and heterogeneity was inevitable. Although rivastigmine and donepezil are both ChEls, their effects on PD dementia are distinct. Only rivastigmine is effective for PDD, and its inhibition of both butyrylcholinesterase and acetylcholinesterase explains its difference from donepezil, which only inhibits acetylcholinesterase. Merging the results from these two ChEls introduces heterogeneity. PD patients were also not uniformly selected. Some researchers have specifically recruited PD patients with falls, whereas others have included general PD patients. Besides, although FOG is the most notorious gait disturbance in $\mathrm{PD}$, only one study assessed the effect of ChEI on PD-FOG by the FOG questionnaire, which demonstrated no significant benefit ${ }^{26}$. Finally, to conduct a gait analysis, the recruited PD patients had to be at least able to walk independently, which excluded PD patients with the most severe gait disturbance and highest risk of fall.

In conclusion, ChEl treatment significantly improved gait variability in PD patients and provided trending benefits in terms of gait speed and fall prevention. Considering the tolerability and cognitive benefits, ChEls may be considered an alternative medical treatment for gait disturbance in PD patients. Future RCTs are warranted to compare the effect between rivastigmine and donepezil and the anatomical origin, either NBM or PPN cholinergic neurons, of gait benefit.

\section{METHODS}

\section{Inclusion criteria}

This study included only randomized controlled trials (RCTs) that investigated the effects of ChEls (including rivastigmine, donepezil, and galantamine) on gait, balance, and fall in patients with idiopathic PD. RCTs were required to clearly report the patient inclusion and exclusion criteria; the process of randomization; method, dosage, and duration of ChEls; and a comprehensive assessment of gait, balance, and fall. This study was registered with PROSPERO (CRD42021254733).

\section{Literature search strategy}

We searched for RCTs published before May 2021 in the PubMed, Embase, and Web of Science databases following the Preferred Reporting Items for Systematic Review and Meta-Analysis (PRISMA) guidelines. The search keywords are as follows: ("Parkinson's disease" [title/abstract] OR "Parkinson disease" [title/abstract]) AND ("cholinesterase inhibitors" [title/ abstract] OR "donepezil" [title/abstract] OR "rivastigmine" [title/abstract] OR "galantamine" [title/abstract]) AND ("gait" [title/abstract] OR "walk" [title/ abstract] OR "balance" [title/abstract] OR "fall" [title/abstract] OR "falls" [title/abstract]). Only studies published in English were included.

\section{Data extraction}

Baseline and outcome data were independently retrieved by two reviewers (J-H.C. and T-W.H.). Furthermore, data on study designs, study population characteristics, and inclusion and exclusion criteria were extracted. Decisions recorded individually by the reviewers were compared, and disagreements were resolved by a third reviewer (C-T.H.).

\section{Outcomes}

The primary outcome was the effect of ChEls on gait parameters, including speed and variability. The secondary outcome was changes in balance and fall. If more than one assessments on balance scale was used in a single study, the more commonly used scale was selected for outcome analysis. 


\section{Appraisal of methodological quality}

Two reviewers (C-T.H. and H-T.H.) independently assessed the methodological quality of each study using the revised risk of bias (version 2.0) method, as recommended by the Cochrane Collaboration. The included studies were scored to determine whether they had a high, medium, or low overall risk of bias. The risk of bias was calculated through the assessment of five domains: bias resulting from the randomization process, bias resulting from deviations from intended interventions, bias resulting from missing outcome data, bias in the measurement of outcomes, and bias in the selection of reported results.

\section{Statistical analysis}

Data were entered and analyzed using Review Manager 5.3 (The Cochrane Collaboration, Oxford, England). A meta-analysis was performed following the PRISMA guidelines. The standard deviation was calculated using the provided confidence interval $(\mathrm{Cl})$ limits, standard errors, or interquartile ranges, where appropriate. The effect sizes of continuous outcomes were reported as the standardized mean difference (SMD). The precision of effect sizes was reported using a $95 \% \mathrm{Cl}$. A pooled estimate of weighted mean difference (WMD) was computed using the DerSimonian and Laird random-effects method. A statistically significant result was indicated by a $p$ value of $<0.05$ or a $95 \% \mathrm{Cl}$ that did not include 1 in the relative risk ratio and 0 in the WMD estimation. Statistical heterogeneity and inconsistency in treatment effects across the studies were evaluated using the Cochrane $Q$-test and $R^{2}$ statistic, respectively. Statistical significance was set at a $p$ value of $<0.10$ for the Cochrane $Q$-test. Statistical heterogeneity across the studies was assessed using the $P^{2}$ statistic, which quantifies the proportion of total outcome variability across studies.

Received: 29 June 2021; Accepted: 4 November 2021; Published online: 25 November 2021

\section{REFERENCES}

1. de Lau, L. M. \& Breteler, M. M. Epidemiology of Parkinson's disease. Lancet Neurol. 5, 525-535 (2006).

2. Lim, S. Y., Fox, S. H. \& Lang, A. E. Overview of the extranigral aspects of Parkinson disease. Arch. Neurol. 66, 167-172 (2009).

3. Jankovic, J. \& Kapadia, A. S. Functional decline in Parkinson disease. Arch. Neurol. 58, 1611-1615 (2001).

4. Celiker, O., Demir, G., Kocaoglu, M., Altug, F. \& Acar, F. Comparison of subthalamic nucleus vs. globus pallidus interna deep brain stimulation in terms of gait and balance; a two year follow-up study. Turk. Neurosurg. 29, 355-361 (2019).

5. Muslimovic, D., Post, B., Speelman, J. D., Schmand, B. \& de Haan, R. J. Determinants of disability and quality of life in mild to moderate Parkinson disease. Neurology 70, 2241-2247 (2008).

6. Fasano, A., Canning, C. G., Hausdorff, J. M., Lord, S. \& Rochester, L. Falls in Parkinson's disease: a complex and evolving picture. Mov. Disord. 32, 1524-1536 (2017).

7. Amboni, M. et al. Prevalence and associated features of self-reported freezing of gait in Parkinson disease: the DEEP FOG study. Parkinsonism Relat. Disord. 21, 644-649 (2015).

8. Amboni, M., Cozzolino, A., Longo, K., Picillo, M. \& Barone, P. Freezing of gait and executive functions in patients with Parkinson's disease. Mov. Disord. 23, 395-400 (2008).

9. Salazar, R. D. et al. Dual tasking in Parkinson's disease: cognitive consequences while walking. Neuropsychology 31, 613-623 (2017).

10. van lersel, M. B., Hoefsloot, W., Munneke, M., Bloem, B. R. \& Olde Rikkert, M. G. Systematic review of quantitative clinical gait analysis in patients with dementia. Z. Gerontol. Geriatr. 37, 27-32 (2004).

11. Tinetti, M. E., Speechley, M. \& Ginter, S. F. Risk factors for falls among elderly persons living in the community. N. Engl. J. Med. 319, 1701-1707 (1988).

12. Yogev-Seligmann, G., Hausdorff, J. M. \& Giladi, N. The role of executive function and attention in gait. Mov. Disord. 23, 329-342 (2008).

13. Woollacott, M. \& Shumway-Cook, A. Attention and the control of posture and gait: a review of an emerging area of research. Gait Posture 16, 1-14 (2002).

14. Kucinski, A., Paolone, G., Bradshaw, M., Albin, R. L. \& Sarter, M. Modeling fall propensity in Parkinson's disease: deficits in the attentional control of complex movements in rats with cortical-cholinergic and striatal-dopaminergic deafferentation. J. Neurosci. 33, 16522-16539 (2013).

15. Sarter, M., Albin, R. L., Kucinski, A. \& Lustig, C. Where attention falls: Increased risk of falls from the converging impact of cortical cholinergic and midbrain dopamine loss on striatal function. Exp. Neurol. 257, 120-129 (2014).
16. Koshy Cherian, A., Kucinski, A., Wu, R., de Jong, I. E. M. \& Sarter, M. Co-treatment with rivastigmine and idalopirdine reduces the propensity for falls in a rat model of falls in Parkinson's disease. Psychopharmacology 236, 1701-1715 (2019).

17. Kucinski, A., de Jong, I. E. \& Sarter, M. Reducing falls in Parkinson's disease: interactions between donepezil and the 5-HT(6) receptor antagonist idalopirdine on falls in a rat model of impaired cognitive control of complex movements. Eur. J. Neurosci. 45, 217-231 (2017).

18. Morris, R. et al. Overview of the cholinergic contribution to gait, balance and falls in Parkinson's disease. Parkinsonism Relat. Disord. 63, 20-30 (2019).

19. Emre, M. et al. Rivastigmine for dementia associated with Parkinson's disease. $N$. Engl. J. Med. 351, 2509-2518 (2004).

20. Ravina, B. et al. Donepezil for dementia in Parkinson's disease: a randomised, double blind, placebo controlled, crossover study. J. Neurol. Neurosurg. Psychiatry 76, 934-939 (2005).

21. Dubois, B. et al. Donepezil in Parkinson's disease dementia: a randomized, double-blind efficacy and safety study. Mov. Disord. 27, 1230-1238 (2012).

22. Sawada, H. et al. Early use of donepezil against psychosis and cognitive decline in Parkinson's disease: a randomised controlled trial for 2 years. J. Neurol. Neurosurg. Psychiatry 89, 1332-1340 (2018).

23. Chung, K. A., Lobb, B. M., Nutt, J. G. \& Horak, F. B. Effects of a central cholinesterase inhibitor on reducing falls in Parkinson disease. Neurology 75, 1263-1269 (2010).

24. Mancini, M. et al. Effects of augmenting cholinergic neurotransmission on balance in Parkinson's disease. Parkinsonism Relat. Disord. 69, 40-47 (2019).

25. Li, Z. et al. Impact of rivastigmine on cognitive dysfunction and falling in Parkinson's disease patients. Eur. Neurol. 74, 86-91 (2015).

26. Henderson, E. J. et al. Rivastigmine for gait stability in patients with Parkinson's disease (ReSPonD): a randomised, double-blind, placebo-controlled, phase 2 trial. Lancet Neurol. 15, 249-258 (2016).

27. Stuart, S. et al. Prefrontal cortex activity and gait in Parkinson's disease with cholinergic and dopaminergic therapy. Mov. Disord. 35, 2019-2027 (2020).

28. Wilson, J., Allcock, L., Mc Ardle, R., Taylor, J.-P. \& Rochester, L. The neural correlates of discrete gait characteristics in ageing: a structured review. Neurosci. Biobehav. Rev. 100, 344-369 (2019).

29. Gaspar, P. \& Gray, F. Dementia in idiopathic Parkinson's disease. A neuropathological study of 32 cases. Acta Neuropathol. 64, 43-52 (1984).

30. Lee, J. E. et al. Exploratory analysis of neuropsychological and neuroanatomical correlates of progressive mild cognitive impairment in Parkinson's disease. $J$. Neurol. Neurosurg. Psychiatry 85, 7-16 (2014).

31. Yogev-Seligmann, G., Giladi, N., Gruendlinger, L. \& Hausdorff, J. M. The contribution of postural control and bilateral coordination to the impact of dual tasking on gait. Exp. Brain Res. 226, 81-93 (2013).

32. Yogev, G. et al. Dual tasking, gait rhythmicity, and Parkinson's disease: which aspects of gait are attention demanding? Eur. J. Neurosci. 22, 1248-1256 (2005).

33. Plotnik, M., Giladi, N., Dagan, Y. \& Hausdorff, J. M. Postural instability and fall risk in Parkinson's disease: impaired dual tasking, pacing, and bilateral coordination of gait during the "ON" medication state. Exp. Brain Res. 210, 529-538 (2011).

34. MacLaren, D. A., Santini, J. A., Russell, A. L., Markovic, T. \& Clark, S. D. Deficits in motor performance after pedunculopontine lesions in rats-impairment depends on demands of task. Eur. J. Neurosci. 40, 3224-3236 (2014).

35. Karachi, C. et al. Cholinergic mesencephalic neurons are involved in gait and postural disorders in Parkinson disease. J. Clin. Invest. 120, 2745-2754 (2010).

36. Bohnen, N. I. et al. History of falls in Parkinson disease is associated with reduced cholinergic activity. Neurology 73, 1670-1676 (2009).

37. Danion, F., Varraine, E., Bonnard, M. \& Pailhous, J. Stride variability in human gait: the effect of stride frequency and stride length. Gait Posture 18, 69-77 (2003).

38. Hausdorff, J. M. et al. Impaired regulation of stride variability in Parkinson's disease subjects with freezing of gait. Exp. Brain Res. 149, 187-194 (2003).

39. Byun, S. et al. Gait variability can predict the risk of cognitive decline in cognitively normal older people. Dement. Geriatr. Cogn. Disord. 45, 251-261 (2018).

40. Schaafsma, J. D. et al. Gait dynamics in Parkinson's disease: relationship to Parkinsonian features, falls and response to levodopa. J. Neurol. Sci. 212, 47-53 (2003).

41. Pieruccini-Faria, F. et al. Gait variability across neurodegenerative and cognitive disorders: results from the Canadian Consortium of Neurodegeneration in Aging (CCNA) and the Gait and Brain Study. Alzheimers Dement. 17, 1317-1328 (2021).

42. Ehgoetz Martens, K. A., Ellard, C. G. \& Almeida, Q. J. Does anxiety cause freezing of gait in Parkinson's disease? PLoS ONE 9, e106561 (2014).

43. Takakusaki, K., Habaguchi, T., Ohtinata-Sugimoto, J., Saitoh, K. \& Sakamoto, T. Basal ganglia efferents to the brainstem centers controlling postural muscle tone and locomotion: a new concept for understanding motor disorders in basal ganglia dysfunction. Neuroscience 119, 293-308 (2003).

44. Park, J. H., Kang, Y. J. \& Horak, F. B. What is wrong with balance in Parkinson's disease? J. Mov. Disord. 8, 109-114 (2015).

45. Wang, J. W. et al. Deep brain stimulation of pedunculopontine nucleus for postural instability and gait disorder after Parkinson disease: a meta-analysis of individual patient data. World Neurosurg. 102, 72-78 (2017). 
46. Allen, N. E., Schwarzel, A. K. \& Canning, C. G. Recurrent falls in Parkinson's disease: a systematic review. Parkinsons Dis. 2013, 906274 (2013).

47. Pieruccini-Faria, F. et al. Gait variability across neurodegenerative and cognitive disorders: results from the Canadian Consortium of neurodegeneration in aging (CCNA) and the Gait and Brain Study. Alzheimer's Dement. 17, 1317-1328 (2021).

48. Delval, A. et al. Gait and attentional performance in freezers under methylphenidate. Gait Posture 41, 384-388 (2015).

49. Seppi, K. et al. Update on treatments for nonmotor symptoms of Parkinson's disease-an evidence-based medicine review. Mov. Disord. 34, 180-198 (2019).

\section{AUTHOR CONTRIBUTIONS}

JH Chen participated the Study conception and design, Data acquisition and analysis, and Draft writing. TW Huang participated the Data acquisition and analysis, Data interpretation, and Manuscript revision. CT Hong participated the study conception and design, data acquisition and analysis, data interpretation, draft writing and manuscript revision. All authors critically revised the manuscript and approved its content before submission. $\mathrm{JH}$ Chen and TW Huang contributed equally in this manuscript.

\section{COMPETING INTERESTS}

The authors declare no competing interests.

\section{ADDITIONAL INFORMATION}

Correspondence and requests for materials should be addressed to Chien-Tai Hong

Reprints and permission information is available at http://www.nature.com/ reprints

Publisher's note Springer Nature remains neutral with regard to jurisdictional claims in published maps and institutional affiliations.

(i) Open Access This article is licensed under a Creative Commons Attribution 4.0 International License, which permits use, sharing, adaptation, distribution and reproduction in any medium or format, as long as you give appropriate credit to the original author(s) and the source, provide a link to the Creative Commons license, and indicate if changes were made. The images or other third party material in this article are included in the article's Creative Commons license, unless indicated otherwise in a credit line to the material. If material is not included in the article's Creative Commons license and your intended use is not permitted by statutory regulation or exceeds the permitted use, you will need to obtain permission directly from the copyright holder. To view a copy of this license, visit http://creativecommons. org/licenses/by/4.0/.

(C) The Author(s) 2021 